\title{
Experimental demonstration of cascaded transmission and all-optical label swapping of orthogonal IM/FSK labelled signal
}

Chi, Nan; Zhang, Jianfeng; Holm-Nielsen, Pablo Villanueva; Xu, Lin; Tafur Monroy, Idelfonso; Peucheret, Christophe; Yvind, Kresten; Christiansen, Lotte Jin; Jeppesen, Palle

\section{Published in:}

Electronics Letters

Link to article, DOI:

10.1049/el:20030425

Publication date:

2003

Document Version

Publisher's PDF, also known as Version of record

Link back to DTU Orbit

Citation (APA):

Chi, N., Zhang, J., Holm-Nielsen, P. V., Xu, L., Tafur Monroy, I., Peucheret, C., Yvind, K., Christiansen, L. J., \& Jeppesen, P. (2003). Experimental demonstration of cascaded transmission and all-optical label swapping of orthogonal IM/FSK labelled signal. Electronics Letters, 39(8), 676 - 678. https://doi.org/10.1049/el:20030425

\section{General rights}

Copyright and moral rights for the publications made accessible in the public portal are retained by the authors and/or other copyright owners and it is a condition of accessing publications that users recognise and abide by the legal requirements associated with these rights.

- Users may download and print one copy of any publication from the public portal for the purpose of private study or research.

- You may not further distribute the material or use it for any profit-making activity or commercial gain

- You may freely distribute the URL identifying the publication in the public portal 
were very stable for both cases. This shows that the PNC output was not seriously corrupted by the laser phase noise as expected [4].

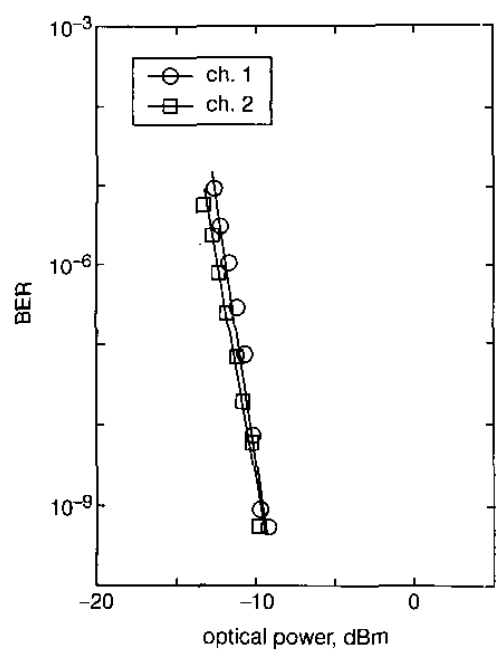

Fig. 4 Measured BERs

Fig. 4 shows the measured BERs after the $25 \mathrm{~km}$-long SMF transmission against received optical power measured ar the input of the $3 \mathrm{~dB}$ optical coupler. The measured BERs for both channel selection were almost the same. BER of less than $10^{-9}$ was achieved when the received optical power was $-10 \mathrm{dBm}$. From the above results, it was experimentally verified that the proposed channel selection scheme is useful for the uplink in ROF systems.

Conclusion: We have proposed the channel selection scheme of mmwave-band SCM ROF signal with optical heterodyne detection, which was performed by tuning the mode interval of the dual-mode local light. It has been shown that the desired channel could be selected without the serious laser-phase-noise and fibre-dispersion effects even if the dual-mode local light was free-running. Moreover, the channel selection of two-channel SCM ROF signal after the $25 \mathrm{~km}$-long SMF transmission has been experimentally verified.

Acknowledgments: T. Kuri would like to thank N. Otani, T. Itabe and T. Iida of the CRL for their encouragement. This research was supported by 'Support system for R\&D activities in infocommunications area' conducted by TAO of Japan.

(C) IEE 2003

Electronics Letters Online No: 20030438

19 Feisuary 2003

DOI: 10.1049/el:20030438

T. Kuri (Communications Research Laboratory, Koganei, Tokyo 184-8975, Japan)

K. Kitayama (Graduate School of Engineering, Osakia University, Suita, Osaka 565-0871, Japan)

\section{References}

1 OKOSHI, T., and KJKUCHF, K.: 'Frequency stabilisation of semiconductor lasers for heterodyne-type optical communication systems', Electron. Lett., 1980, 16, pp. 179-181

2 KIKUCHI, K., and KATOH, K.: 'Optical heterodyne receive,: for selecting densely frequency division multiplexed signals', Electron. Lett., 2002, 38, pp. 283-285

3 GROSS, R., OLSHANSKY, R., and SHUMIDT, M.: 'Coherent FM-SCM system using DFB lasers and a phase noise canceling circuit', IEEE Photonics Technol. Lett., 1988, 2, (1), pp. 66-68

4 KURI, T, and KITAYAMA, K.: 'Optical heterodyne detection of millimeterwave-band radio-on-fiber signal with remote dual-mode local light source', IEEE Trans. Microw. Theory Tech., 2001, 49, (10), pp. 2025-2029

5 KURI, T., KITYAMA, K., STÖHR, A, and OGAWA, Y.: 'Fiber-optic millimeterwave downlink system using $60 \mathrm{GHz}$-band external modulation', J. Lightwave Technol., 1999, 17, (5), pp. 799-806

\section{Experimental demonstration of cascaded transmission and all-optical label swapping of orthogonal IM/FSK labelled signal}

Nan Chi, Jianfeng Zhang, P.V. Holm-Nielsen, Lin Xu, I.T. Monroy, C. Peucheret, K. Yvind, L.J. Christiansen and P. Jeppesen

\begin{abstract}
A network node is demonstrated with two-hop transmission and alloptical label swapping based on a Mach-Zehnder semiconductor optical amplifier interferometer and electroabsorption modulator of a two-level optically labelled signal using an orthogonal IM/FSK modulation format with an overall power penalty of less than $2 \mathrm{~dB}$.
\end{abstract}

Introduction: All-optical label swapping is an attractive technique for implementing packet routing and forwarding functions independently of IP packet length and payload bit rate [1]. Combined intensity modulation/frequency-shift keying (IM/FSK) or intensity modulation/phase-shift keying (IM/DPSK) optical labelling has been proposed as a competing scheme to sub-carrier multiplexed optical labelling due to its compact spectrum, simple label swapping and remarkable scalability to high bit rates [2-4]. Although we have earlier demonstrated successful single hop transmission of an IM/FSK labelled signal generated by a distributed feedback (DFB) laser with an integrated electroabsorption modulator (EAM) [3], the feasibility of all-optical label swapping and multi-hop transmission of this IM/FSK labelling scheme needs to be verified.

In this Letter, we present the latest experimental investigation of the complete functionality of a real network node with two-hop transmission and all-optical label swapping of an optically IM/FSK labelled signal. Simultaneous FSK label erasure and $2 \mathrm{R}$ regeneration are successfully achieved in a monolithically integrated Mach-Zehnder semiconductor optical amplifier (MZ-SOA). We atso demonstrate for the first time FSK label insertion bascd on an EAM through all-optical conversion of the $10 \mathrm{Gbit} / \mathrm{s}$ intensity modulated payload to a new wavelength. Propagation over two transmission spans (50 and $44 \mathrm{~km}$ SMF) separated by a network node including label removal, $2 \mathrm{R}$ regeneration, and label reinsertion functionalities, is shown to result in less than $2 \mathrm{~dB}$ power penalty for both the $10 \mathrm{Gbit} / \mathrm{s} \mathrm{IM}$ payload and the $312 \mathrm{Mbit} / \mathrm{s}$ FSK modulated label.

Architecture of all-optical packet switched network based on orthogonal IM/FSK labelling: At the ingress edge router the incoming IP packets are assigned two-level optical labels, i.e. the wavelength of the signal carrier ( $i$ label) and the FSK label, orthogonally modulated to the IM payload. The packet switched network architecture requires these two-level optical labels to be swapped during the routing process in order to build up an appropriate optical path along the transmission fibre network, as shown in Fig. 1.

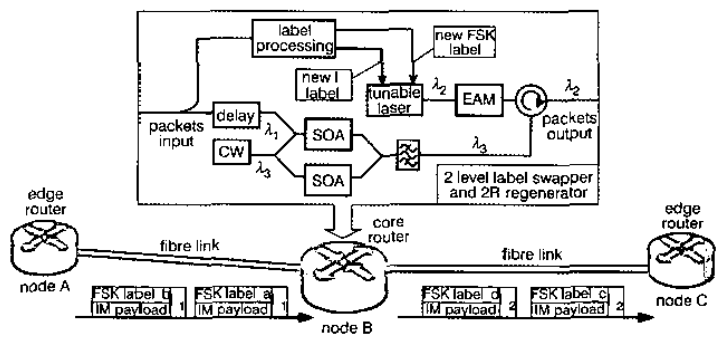

Fig. 1 System architecture for orthogonal IM/FSK labelling

The core nodes perform routing and forwarding operations based on the information retrieved from the optical label. They also perform label swapping with wavelength conversion to ensure that packets reach their destination. To perform label swapping, a fraction of the incoming signal is tapped for opto-electronic label processing. The remaining part of the signal is input to an MZ-SOA for label removal and $2 \mathrm{R}$ regeneration. Replacing the label is easy because the FSK label information is lost during the wavelength conversion in the MZ-SOA. Therefore only the payload information will be copied onto the output wavelength of the MZ-SOA, which will then be used as the pump signal to the EAM. After setting the new $\lambda$ label by accordingly setting the 
wavelength of the tunable laser and inserting the new FSK label, the FSK modulated signal is injected into the EAM as the probe signal for frequency-modulation maintaining wavelength conversion. After these cascaded processes, both the $i$ label and the FSK label are swapped and the packet is ready for the next hop transmission.

Experimental setup and results: The experimental setup is shown in Fig. 2. The optical FSK modulation can be achieved simply by directly modulating the electrical current of a DFB laser $(1549.2 \mathrm{~nm})$. However, the drive current variation always results in a simultaneous intensity modulation of the emitted light, which will obviously cause a detrimental effect on the IM payload. To remove the intensity variation at the output of the laser, the inverse electrical data is injected into the integrated EA modulator with appropriate time delay and modulation voltage. In this way, a constant amplitude optical FSK signal at $312 \mathrm{Mbit} / \mathrm{s}$ (PRBS $2^{7}-1$ ) is generated. The payload information at $10 \mathrm{Gbit} / \mathrm{s}$ (PRBS $2^{9}-1$ ) is added by a following Mach-Zehnder modulator, thus producing an optically IM/FSK labelled signal. It should be noted that a limited IM extinction ratio is necessary for the FSK label detection, but on the other hand this low extinction ratio will deteriorate the payload detection. Therefore in our experiment a compromise value of $4.3 \mathrm{~dB}$ is selected for the extinction ratio of the IM payload.

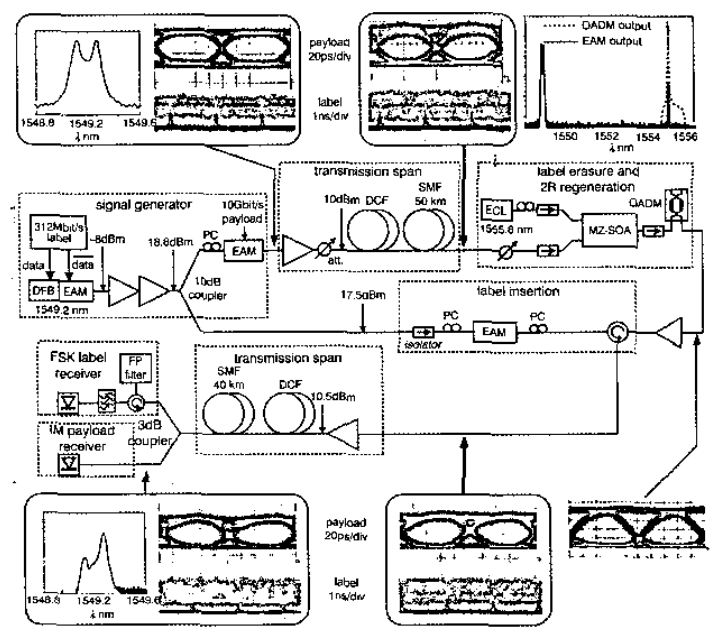

Fig. 2 Experimental setup

The first hop consists of $50 \mathrm{~km}$ singlemode fibre (SMF) with matching length of dispersion compensating fibre (DCF). The dispersion of the SMF and the DCF is $16.9 \mathrm{ps} / \mathrm{nm} / \mathrm{km}$ and $-100 \mathrm{ps} / \mathrm{nm} / \mathrm{km}$, respectively. Our simulation results and experimental investigation identically reveal that a pre-compensation scheme has better performance than a post-compensation scheme. Hence pre-compensated fibre spans are chosen for both fibre links. After this first stage transmission the optically labelled signal is input to the MZ-SOA (Alcatel 1901ICM) for the label erasure and $2 \mathrm{R}$ regeneration. A tunable external cavity laser at $1555.8 \mathrm{~nm}$ is used as CW input for the MZ-SOA. Very good label erasure and $2 \mathrm{R}$ regeneration can be achieved by the MZ-SOA. Owing to its nonlinear transfer function, the extinction ratio of the converted signal is greatly improved to $12.9 \mathrm{~dB}$, which leads to a $2 \mathrm{~dB}$ enhancement for the receiver sensitivity. The regenerated payload is then fed to the EAM as the pump signal for the label insertion process, where the extinction ratio of the label-renewed signal will be adapted to the required value.

The advantage of using the EAM for label reinsertion is the negligible frequency chirp induced by EAM-based wavelength conversion, which is extremely desirable in our orthogonal IM/FSK labelling scheme [5]. The EAMs used in our experiment were kindly provided by GIGA, an Intel company. The initial FSK signal is split into two parts with one of them acting as the second label source for the label insertion module. The nonlinear transfer function of the MZ-SOA will be beneficial to the output extinction ratio at the cost of some amplitude jitter and eye diagram distortion. This can be seen in the eye diagram recorded at the output of the MZ-SOA shown in Fig. 2. Another advantage of the label swapping based on cascaded MZ-SOA and EAM is that this amplitude jitter can be compensated for by the nonlinear absorption and saturable absorption of the EAM. As evidence a very clear eye diagram with negligible distortion was obtained when carrying out cascaded conversion of a pure IM signal. It should be noted that the noise imposed onto the EAM output eye diagram (see Fig. 2) is due to the non-optimal FSK intensity. As mentioned earlier, a limited extinction ratio is obligatory in the orthogonal labelling scheme. This relatively low extinction ratio can be easily accomplished by adjusting the reverse bias of the EAM. The output signal of the label swapper has an extinction ratio of $4.9 \mathrm{~dB}$. The second hop includes $44 \mathrm{~km} \mathrm{SMF}$ and $6 \mathrm{~km}$ DCF. At the receiver, the frequency discrimination for FSK demodulation is achieved by two optical filter stages providing more than $15 \mathrm{~dB}$ suppression ratio between the two FSK tones.

Fig. 3 shows the BER curves in the back-to-back case, after the first hop, after the label swapper and after the second hop. The inset shows the pattern for both the payload and the label as detected after two-hop transmission including label swapping. Clearly some payload information is superimposed onto the label after the FSK demodulation, however the eye is still open and allows error-free detection. The cascaded transmission and label swapping result in $1.9 \mathrm{~dB}$ power penalty for the payload and $1.8 \mathrm{~dB}$ penalty for the label.

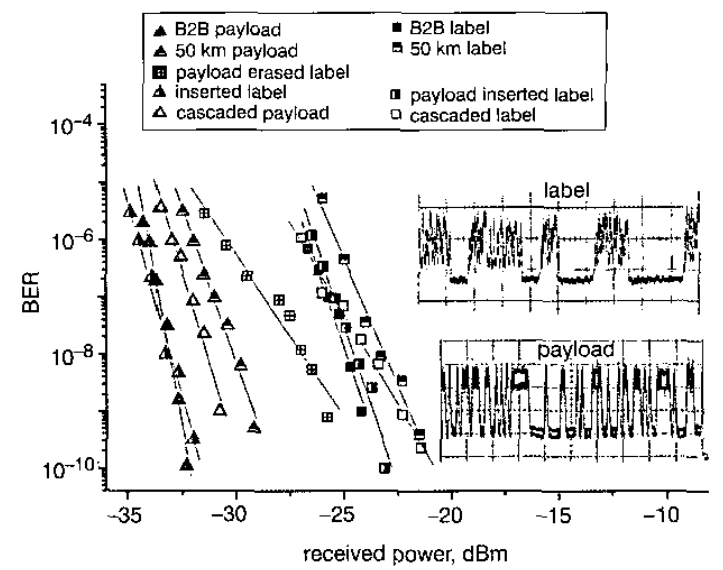

Fig. 3 Measured BER results for payload and label

Inset: Reccived pattem of payload and label after two-hop transmission and label swapping

Conclusion: We have experimentally demonstrated the full functionality of a network node performing all-optical label swapping of a two-level optically labelled signal using orthogonal IM/FSK modulation format, two-hop transmission and $2 \mathrm{R}$ regeneration. The power penalty of the cascaded transmission and label swapping was shown to be below $2 \mathrm{~dB}$.

Acknowledgment: This work is performed within the framework of the IST STOLAS (Switching Technologies for Optically Labeled Signals) project supported by the EU Commission.

(C) IEE 2003

Electronics Letters Online No: 20030425

24 February 2003

DOI: 10.1049/el:20030425

Nan Chi, Jianfeng Zhang, P.V. Holm-Nielsen, Lin Xu, C. Peucheret, K. Yvind, L.J. Christiansen and P. Jeppesen (COM, Technical University of Denmark, Building 345v, DK-2800 Kgs., Lyngby, Denmark)

E-mail: nc@com.dtu.dk

I.T. Monroy (COBRA Research Institute, Eindhoven University of Technology, The Netherlands)

\section{References}

I BLUMENTHAI, D.J, OLSSON, B.E., ROSSI, G., DIMMICK, T.E., RAU. L., MASANOVIC, M., LAVROVA. O., DOSHI, R., JERPHAGNON, O, BOWERS. J.E., KAMAN. V, COLDREN, L.A., and BARTON, J.: 'All-optical label swapping networks and technologies', J. Lightwave Technol., 2000, 18, (12), pp. 2058-2075

2 KOONEN, T, MORTHIER, G., JENNEN, J., DE WAARDT, H, and DEMEFSTER, P: 'Optical packet routing in IP-over-WDM networks deploying two-level optical labeling'. Proc. of European Conf. on Optical Communication, ECOC'01, Amsterdam, The Netherlands, 2001, Vol. 4, pp. 608-609, Paper Th.L.2.1 
3 ZHANG, J., CHI. N., HOLM-NIFLSEN, P.,., PEUCHERET, C, and JEPPESEN, P.: 'A novel method for optical subcarrier label generation'. Proc. of Optical Fiber Communication Conf., OFC'2003, Atlanta, GA, USA, 2003, Paper FD5

4 CHI, N., CARLSSON, B., ZHANG, 3., HOLM-NIELSEN, P.V., PEUCHERET, C., and JEPPESEN, P.: 'Transmission performance of all-optically labeled packets using ASK/DPSK orthogonal modulation'. Proc. IEEE Laser and Electro-Optics Society Annual Meeting, LEOS'2002, Glasgow, Scotland, UK, 2002, Paper MF3

5 XU, L., OXENLøWE, L.K., CHI, N., ROMSTAD, F.P, YVIND, K., MøRK, J., JEPPESEN. $P$., HOPPE, $K$, and HANBERG, $J$. 'Bandwisth and chirp characterisation of wavelength conversion based on electroabsorption modulators'. Proc. of European Conf. on Optical Communication, ECOC'02, Copenhagen, Denmark, 2002, Vol. 3, Paper P1.26

\section{Mitigation of optical crosstalk penalty in photonic cross-connects using forward error correction}

\author{
V. Kaman, X. Zheng, C. Pusarla, A.J. Keating, \\ R.J. Helkey and J.E. Bowers
}

The use of forward error correction in all-optical networks for reducing the impact of dynamic crosstalk in photonic cross-connects based on three-dimensional microelectromechanical systems (3DMEMS) is described. Error-free operation is achieved for coherent crosstalk levels up to $-16 \mathrm{~dB}$, which corresponds to a $13.5 \mathrm{~dB}$ coherent input signal dynamic range, using a RS(255/239) ReedSolomon error correcting code with a coding gain of $6 \mathrm{~dB}$ in a $288 \times 288$ non-blocking 3D-MEMS switch

Introduction: Dense wavelength division-multiplexed fhotonic networks with all-optical (OOO) nodes for metro and long-haul applications have recently been demonstrated for network efficiency and significant cost savings by the removal of optical-electrical-optical transponders at each switching node [1]. With their potential for realising large-scale and compact photonic cross-connects (PXC), free-space three-dimensional microelectromechanical systems (3DMEMS) based optical switches have been proven as the leading technology for these $00 O$ switching nodes, with switch sizes exceeding 200 ports $[2,3]$. Photonic networks based on 000 switching nodes can, however, suffer from the addition of leakage crosstalk from adjacent wavelengths in a cascade of wavelength multiplexers [4] as well as possible dynamic crosstalk arising as an input mirror scans instantaneously over an unintended output mirror in 3D-MEMS switches [5]

Several transmission impairments, such as noise accumulation in optically amplified long-haul systems [6] and polarisation mode dispersion [7], have been alleviated by using forward error correction (FEC). More recently, the use of FEC has been proposed for in-band coherent crosstalk [8]. In this Letter, we investigate the benefit of using FEC against instantaneous dynamic crosstalk in photonic networks based on 3D-MEMS optical switching nodes. Our results simulate levels beyond expected worst-case optical crosstalk and can be extended to other sources of crosstalk such as in a cascade of wavelength multiplexers or filters $[1,4]$

Photonic cross-connect characteristics: The 288-port PXC system consists of a 3D-MEMS based optical core switch and auxiliary input and output $2 \times 2$ optical switches for 1:1 protection as well as optical taps for power monitoring and mirror control. The light from an input fibre is collimated and incident on a MEMS mirror which can deflect light onto any of the output MEMS mirrors. The output mirror then aligns the optical beam onto a particular output collimator, and the path loss is minimised by optimising the mirror angles. The measured non-blocking core switch and PXC system median losses are 1.4 and $4.3 \mathrm{~dB}$ at $1310 \mathrm{~nm}$, respectively. The extra loss in the PXC is mainly due to the $2 \times 2$ protection switches and the optical tap couplers. The PXC also has a wide transparent optical bandwidth from 1260 to $1625 \mathrm{~nm}$ with a maximum loss variation of $1.5 \mathrm{~dB}$. Static channel isolation, given by the ratio of output power to input power for two ports not in a connection, is below $-60 \mathrm{~dB}$ for input and output ports adjacent to the signal path. Isolation for non-adjacent portsi is typically better than $-80 \mathrm{~dB}$, so the total crosstalk from a fully loaded system is dominated by adjacent ports. Dynamic crosstalk occurs when an input mirror $S_{\text {in }}$, and output mirror $S_{\text {out }}$ are in a connection, and an adjacent input mirror $X_{\text {in }}$ is pointed to output minor $S_{\text {out }}$ when moving $X_{\text {in }}$ from $X_{\text {out } 1}$ to $X_{\text {out } 3}$, as shown in Fig. 1. By manually pointing $X_{\text {in }}$ to $S_{\text {out }}$ while $S_{\text {out }}$ was optimised to $S_{\text {in }}$, the channel isolation was measured to be $-35 \mathrm{~dB}$, although typical isolation is better than $-40 \mathrm{~dB}$.

Results: The experimental setup is shown in Fig. 1. The two $9.95328 \mathrm{Gbit} / \mathrm{s}$ complementary outputs from a bit crror rate tester (BERT) with pattern lengths of $2^{31}-1$ were synchronously fed into the $\mathrm{RS}(255 / 239) \mathrm{FEC}$ encoder. A $1553.3 \mathrm{~nm}$ optical signal was then modulated with the FEC-encoded $10.66 \mathrm{Gbit} / \mathrm{s}$ data stream using a Mach-Zehnder modulator (MOD). A $3 \mathrm{~dB}$ coupler split the output signal and one arm, with $0.5 \mathrm{dBm}$ of power, was directly connected to the main optical signal path in the PXC (mirror $S_{\text {in }}$ to $S_{\text {out }}$ ) with a loss of $5.5 \mathrm{~dB}$. The other arm of the splitter was fed into an erbium-doped optical amplifier (EDFA), which was followed by a variable optical attenuator (VOA) and a polarisation controller (PC) before it was input to the adjacent port (mirror $X_{\text {in }}$ ) of the main signal path. An optically amplified receiver was used at the output of the PXC before the $10.66 \mathrm{Gbit} / \mathrm{s}$ signal was FEC-decoded to $9.95328 \mathrm{Gbit} / \mathrm{s}$ for bit error rate (BER) measurements.

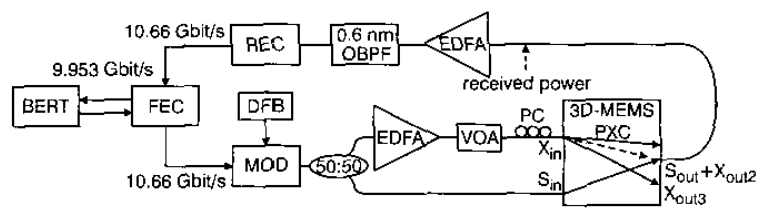

Fig. $110 \mathrm{Gbit} / \mathrm{s}$ experimental setup for dynamic crosstalk experiment DFB: distributed feedback laser; OBPF: optical bandpass filter; REC: receiver

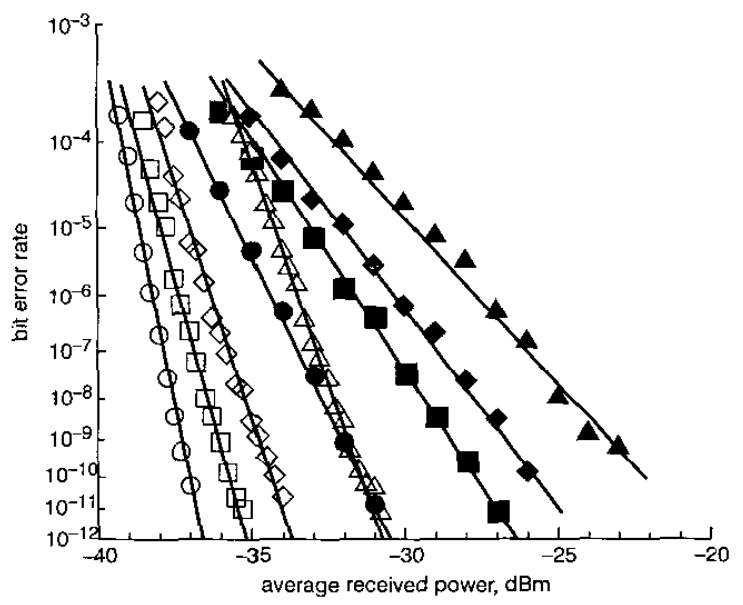

Fig. 2 Bit error rate curves at 10 Gbit/s with no, $-22.5,-20$, and $-17.5 \mathrm{~dB}$ crosstalk

Filled and cmpty symbols denote with and without FEC operation, respectively - Ono crosstalk

口 $-22.5 \mathrm{~dB}$ crosstalk

$\diamond>-20 \mathrm{~dB}$ crosstalk

$\triangle-17.5 \mathrm{dBm}$ crosstalk

Fig. 2 shows the BER results with and without FEC encoding. In these measurements, the channel isolation was held statically at the worst-case value of $-35 \mathrm{~dB}$ experienced during switching while the crosstalk channel input power was set to various levels (no power, 7.5, 10 , and $12.5 \mathrm{dBm}$ ). The signal input power was $0.5 \mathrm{dBm}$ resulting in $-5 \mathrm{dBm}$ output power and a crosstalk $X_{\text {out }} / S_{\text {out }}$ of $0,-22.5,-20$, and $-17.5 \mathrm{~dB}$, respectively. The $\mathrm{PC}$ was used to align the polarisations of the two signals for maximum errors. Without crosstalk, a BER of $10^{-12}$ was obtained for an average received power of $-30.5 \mathrm{dBm}$ without FEC and $-36.5 \mathrm{dBm}$ with FEC. As the interfering signal level was increased to $12.5 \mathrm{dBm}$ to cause a coherent crosstalk level of $-17.5 \mathrm{~dB}$, the power penalty incurred with no FEC is more than $10 \mathrm{~dB}$ while FEC enables the same receiver sensitivity of $-30.5 \mathrm{dBm}$ that was achieved without crosstalk and without FEC. 